\title{
Investigation of type 1 diabetes and coeliac disease susceptibility loci for association with juvenile idiopathic arthritis
}

\author{
Anne Hinks, ${ }^{1}$ Paul Martin, ${ }^{1}$ Edward Flynn, ${ }^{1}$ Steve Eyre, ${ }^{1}$ Jon Packham, ${ }^{2}$ Anne Barton, ${ }^{1}$; \\ Childhood Arthritis Prospective Study (CAPS), UKRAG Consortium, BSPAR study group, \\ Jane Worthington, ${ }^{1}$ Wendy Thomson, ${ }^{1}$
}

\begin{abstract}
- Additional data
(supplementary table) are published online only. To view these files please visit the journal online (http://ard.bmj. com).

${ }^{1}$ Arthritis Research UK Epidemiology Unit, The University of Manchester, Manchester, UK

${ }^{2}$ Haywood Hospital, University Hospital of North Staffordshire, Stoke on Trent, Staffordshire, ST4 7LN.
\end{abstract}

\section{Correspondence to} Dr Anne Hinks, Arthritis Research UK Epidemiology Unit, Manchester Academic Health Science Centre, Stopford Building, The University of Manchester, Manchester M13 9PT, UK;

anne.hinks@manchester.ac.uk

Accepted 25 June 2010

Published Online First 20th July 2010

\begin{abstract}
Background There is strong evidence suggesting that juvenile idiopathic arthritis (JIA) shares many susceptibility loci with other autoimmune diseases. Objective To investigate variants robustly associated with type 1 diabetes (T1D) or coeliac disease (CD) for association with JIA.

Methods Sixteen single-nucleotide polymorphisms (SNPS) already identified as susceptibility loci for T1D/ CD were selected for genotyping in patients with JIA $(n=1054)$ and healthy controls $(n=3129)$. Genotype and allele frequencies were compared using the CochraneArmitage trend test implemented in PLINK.

Results One SNP in the LPP gene, rs1464510, showed significant association with JIA ( $p_{\text {trend }}=0.002,0 R=1.18$, 95\% Cl 1.06 to 1.30). A second SNP, rs653178 in ATXN2, also showed nominal evidence for association with JIA ( $p_{\text {trend }}=0.02, O R=1.13,95 \% \mathrm{Cl} 1.02$ to 1.25$)$. The SNP, rs17810546, in IL12A showed subtype-specific association with enthesitis-related arthritis (ERA) subtype $\left(p_{\text {trend }}=0.005,0 R=1.88,95 \% \mathrm{Cl} 1.2\right.$ to 2.94$)$.

Conclusions Evidence for a novel JIA susceptibility locus, LPP, is presented. Association at the SH2B3/ ATXN2 locus, previously reported to be associated with JIA in a US series, also supports this region as contributing to JIA susceptibility. In addition, a subtypespecific association of IL12A with ERA is identified. All findings will require validation in independent JIA cohorts.
\end{abstract}

\section{INTRODUCTION}

In the past few years huge advances have been made in the understanding of the genes contributing to autoimmune disease susceptibility. It is also becoming clear that, despite the apparent clinical and phenotypic differences of the autoimmune diseases, they share a number of genetic risk factors. ${ }^{1}$ Of note, the genetic overlap between type 1 diabetes (T1D), coeliac disease (CD) and rheumatoid arthritis (RA) ${ }^{2} 3$ (Eyre et al, submitted).

Juvenile idiopathic arthritis (JIA) is the most common chronic rheumatic disease of childhood. It is defined as chronic inflammation of the synovial joints, with unknown aetiology, that starts before the age of 16 and persists for at least 6 weeks. Familial clustering of other autoimmune diseases in JIA families is well established, with an increased prevalence of autoimmunity in first- and seconddegree relatives of patients. ${ }^{4}$

There is already strong evidence suggesting that JIA shares many susceptibility loci with other autoimmune diseases such as RA, T1D and multiple sclerosis. ${ }^{5}{ }^{6}$ Therefore, the aim of this study was to test single-nucleotide polymorphisms (SNPs) robustly associated with $\mathrm{T} 1 \mathrm{D}$ or $\mathrm{CD}$ in a large cohort of patients with JIA and controls to investigate the overlap between these diseases.

\section{METHODS \\ Subjects}

DNA was available for 1054 UK Caucasian patients with JIA (332 men, 715 women) as previously described. ${ }^{6}$ JIA cases were classified according to the International League of Associations for Rheumatology (ILAR) criteria ${ }^{7}$ (online supplementary table 1).

Three thousand one hundred and twenty-nine healthy UK Caucasian control DNA samples were available as described previously. ${ }^{8}$ All individuals were recruited with ethical approval and provided informed consent (North-West Multi-Centre Research Ethics Committee (MREC 99/8/84) and the University of Manchester Committee on the Ethics of Research on Human Beings).

\section{SNP selection}

SNPs that showed robust evidence for association $\left(p<5 \times 10^{-7}\right)$ with T1D and/or $\mathrm{CD}^{2}$ and had not been investigated previously were identified. In total 16 SNPs were selected for genotyping.

\section{Genotyping}

All SNPs were genotyped, in UK JIA cases and controls, using the Sequenom iPlex MassARRAY platform according to manufacturers instructions (Sequenom, San Diego, California, USA, http:// www.sequenom.com, accessed 9 July 2010). A $90 \%$ sample quality control rate and 90\% SNP genotyping success rate was imposed on the analysis.

\section{Statistical analysis}

Power calculations were performed using QUANTO based on the effect sizes reported in previous studies of these SNPs in other autoimmune diseases. Calculations assumed a logadditive model and an $\alpha$ value corrected for the number of SNPs analysed. Genotype and allele frequencies were compared between cases with JIA and controls using PLINK. ${ }^{9}$ Comparisons were expressed as ORs and their 95\% CIs as well as the Cochrane-Armitage test for trend. JIA is a phenotypically heterogeneous disease and can be 


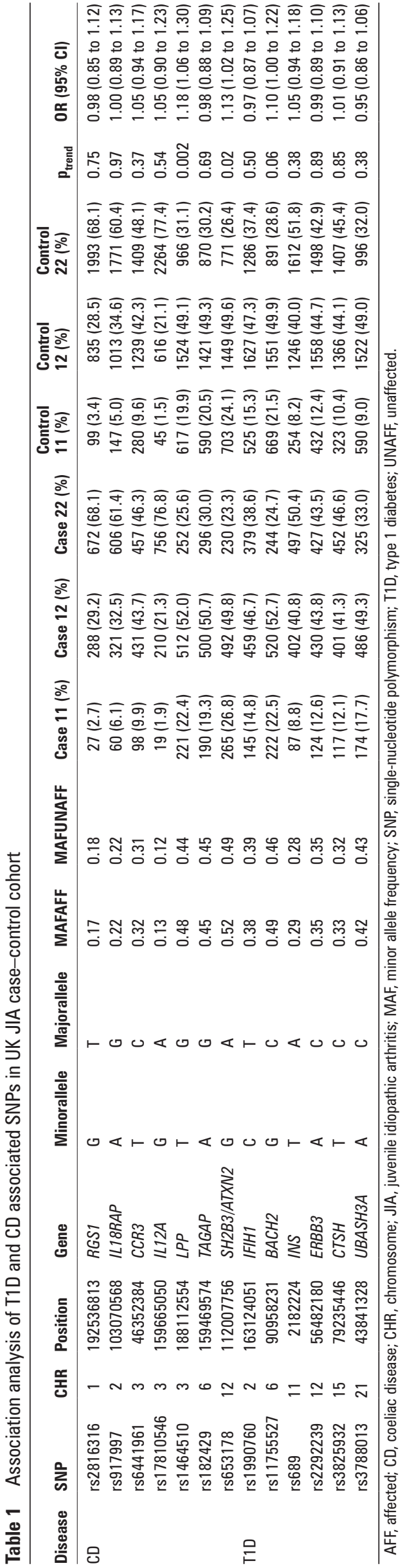

classified into more clinically homogeneous diseases using the ILAR classification criteria. ${ }^{7}$ We examined whether there was evidence of a difference in allele frequencies between the seven ILAR subtypes. Differences between subtypes were assessed using $\chi^{2}$ tests on the $7 \times 2$ tables. Only if a significant difference was detected was stratification analysis by ILAR subtype performed and ORs and 95\% CIs calculated for the subtypes.

\section{RESULTS}

Genotype counts for all SNPs were in Hardy-Weinberg equilibrium $(p>0.05)$ in the control cohort. Three SNPs (rs12708716, rs45450798 and rs229541) failed SNP quality control thresholds and were excluded from further analysis, leaving 13 SNPs for analysis. A Bonferroni correction of 13 was applied to correct for the number of loci studied, resulting in a p value threshold of 0.004 for claims of significance (table 1).

One SNP in the LPP gene associated with CD, rs1464510, showed significant association with JIA at the corrected threshold ( $\mathrm{p}_{\text {trend }}=0.002, \mathrm{OR}=1.18,95 \%$ CI 1.06 to 1.30$)$ (table 1 ). A second SNP, rs653178, in the ATXN2 gene associated with CD, was nominally associated with JIA ( $\mathrm{p}_{\text {trend }}=0.02, \mathrm{OR}=1.13,95 \%$ CI 1.02 to 1.25 ) but this was not statistically significant after correction for multiple testing $\left(p_{\text {trend-corrected }}=0.26\right)$.

All SNPs were examined for differences in allele frequencies across the ILAR subtypes. The SNP, rs17810546, in IL12A associated with $\mathrm{CD}$, showed a significant allele frequency difference $(p=0.03)$ between the subtypes and therefore this SNP was reanalysed stratifying by ILAR subtype. The enthesitis-related arthritis (ERA) subtype showed strong association with IL12A $\mathrm{SNP}\left(\mathrm{p}_{\text {trend }}=0.005, \mathrm{OR}=1.88,95 \%\right.$ CI 1.2 to 2.94$)$. There was no evidence for association with any other subtype.

\section{DISCUSSION}

The emerging overlap of autoimmune disease susceptibility loci makes many of the newly discovered T1D and CD loci appealing candidates for investigation as possible JIA susceptibility loci. There is already some evidence for the overlap of JIA and T1D susceptibility loci, with confirmed association of genes such as the major histocompatibility complex, PTPN22, IL2RA, IL2/21 region and CCR5 with both diseases. ${ }^{5}$ 10-12

By investigating confirmed CD and T1D loci in JIA we have identified a novel locus conferring susceptibility to JIA. This SNP lies within the Lim domain containing preferred translocation partner in lipoma ( $L P P)$ gene. SNPs in $L P P$ show validated association with CD. ${ }^{13} 14$ Interestingly, the rs1464510 SNP does not appear to be associated with $\mathrm{T}^{2} \mathrm{D}^{2}$ and studies in RA show conflicting results: in one large UK population study, no association was detected ( $\mathrm{p}_{\text {trend }}=0.82, \mathrm{OR}=1.01,95 \%$ CI 0.94 to 1.08 ) (Eyre et al, submitted) while in a Dutch population, modest evidence for association was reported $(p=0.012, O R=1.14,95 \%$ CI 1.03 to 1.26$)^{3}$

$L P P$ has a number of known functions; it is important in cell migration, cell adhesion and acts as a versatile scaffolding and adaptor protein. ${ }^{15}$ Recently, LPP was identified as a substrate of the protein-tyrosine phosphatase $1 \mathrm{~B}$, a negative regulator of multiple signalling pathways downstream of receptor tyrosine kinases and functionally linked to Ras signalling. ${ }^{16}$ Further work will be required to determine which of the multiple potential pathways with which the protein interacts is affected by the variants that predispose to autoimmune disease.

We also found weak evidence for association of a SNP, rs653178, in the SH2B3/ATXN2 gene region with JIA, though it did not remain significant after correction for multiple testing. 
The SNP lies within intron 1 of the ATXN2 gene which lies adjacent to the $S H 2 B 3$ gene. This SNP is in complete linkage disequilibrium $\left(\mathrm{r}^{2}=1\right)$ with the SNP, rs3184504, in $S H 2 B 3$, and both SNPs show validated association with CD. ${ }^{13}{ }^{14}$ rs3184504 has been also been associated with both RA and CD in a Dutch population. ${ }^{3}$ The $S H 2 B 3$ rs3184504 variant is a non-synonymous SNP, R262W, located in exon 3 of the gene. SH2B3 encodes the T-cell adapter protein Lnk, which regulates T-cell receptor-, growth factor- and cytokine receptor-mediated signalling. It has been shown to be a negative regulator of tumour necrosis factor $\alpha$ signalling in human endothelial cells. ${ }^{17}$ Another SNP, rs17696736, within the adjacent gene c12orf30, has also been reported to be associated with JIA in a US case-control cohort. ${ }^{18}$ There is only modest linkage disequilibrium between that SNP and the SNP tested in our study $\left(r^{2}=0.5\right)$. Hence, both may simply be markers of an, as yet, un-genotyped causal variant or there may be independent effects in the region.

We also found that the SNP, rs17810546, which lies 5' to the IL12A gene, showed differences in allele frequencies across the ILAR subtypes and this was driven by a strong association with ERA. This is a subgroup comprising mainly male, HLA-B27positive patients and it is characterised by the presence of enthesitis and arthritis. It may progressively involve the sacroiliac joints and spine, with symptoms similar to ankylosing spondylitis. It is important to note that the numbers in this subgroup are small ( $\mathrm{n}=61$ ) so this finding should be interpreted with caution and will require validation in an independent cohort. To date the IL12A gene has not been associated with ankylosing spondylitis.

Although none of the other SNPs studied showed significant association with JIA, we cannot unequivocally rule out association for many of these loci. This is owing to the limited power in this study to detect some of the loci that were identified in the studies for CD and T1D, which had much larger sample sizes. We had $>80 \%$ power to detect an effect at RGS1, IL18RAP, IL12A, INS and ERBB3 and therefore feel more confident that the SNPs tested at those loci are not associated with JIA. OR plots for $C D$ and T1D susceptibility genes across the different autoimmune diseases, JIA, RA, T1D and CD, are shown in figures 1 and 2, respectively.

In conclusion we have identified novel association of the $L P P$ gene and show weak but confirmatory association of the $A T X N 2 / S H 2 B 3 / c 120 r f 30$ region with JIA in a UK cohort. In addition, we report an ERA subtype-specific association with the IL12A gene. Investigation of all these loci in independent JIA case-control cohorts followed by meta-analysis will be required to confidently confirm or refute these as JIA susceptibility loci. Nonetheless, the approach of targeting variants associated with other autoimmune diseases is already yielding insights into the genetic complexity underlying susceptibility to this serious childhood disease.

Childhood arthritis prospective study (CAPS) group Eileen Baildam, Lynsey Brown, Joanne Buckley, Alice Chieng, Joyce Davidson, Michael Eltringham, Helen Foster, Mark Friswell, Janet Gardner-Medwin, Paul Gilbert, Kimme Hyrich, Julie Jones, Sham Lal, Mark Lay, Carol Lydon, Alexandra Meijer, Vicki Price, Jane Sim, Maureen Todd, Peter Ward, Lucy Wedderburn.

UKRAG Consortium Stephen Eyre, Anne Hinks, John Bowes, Gisela Orozco, Edward Flynn, Paul Martin, Wendy Thomson, Anne Barton, Jane Worthington: Arthritis Research UK Epidemiology Unit, Manchester Academic Health Science Centre, Stopford Building, The University of Manchester, Manchester, UK; Stephen Martin, James I Robinson, Ann W Morgan, Paul Emery: Leeds Institute of Molecular Medicine, Section of Musculoskeletal Disease, University of Leeds, Leeds LS9 7TF, UK; Anthony G Wilson: School of Medicine and Biomedical Sciences, The University of Sheffield, Sheffield S1c0 2JF, UK; Sophia Steer: Clinical and Academic Rheumatology, Kings College Hospital NHS Foundation Trust, Denmark Hill, London SE5 9RS, UK; Lynne Hocking, David M Reid: Bone Research Group, Department of Medicine and

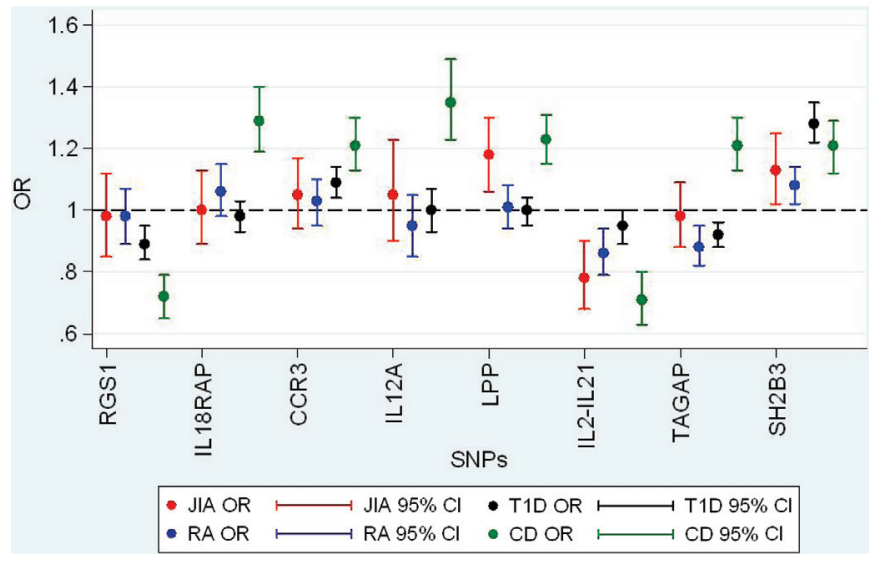

Figure 1 Odds ratio (OR) plots for juvenile idiopathic arthritis (JIA), rheumatoid arthritis (RA), type 1 diabetes (T1D) and coeliac disease (CD) for confirmed CD loci. Data for T1D and CD from Smyth et $a l_{,}{ }^{2}$ for RA from Barton et al ${ }^{19} 20$ and Eyre et al, submitted, for JIA additional data from Hinks et $a l^{56}$ and Hinks et al, 2009 in press.

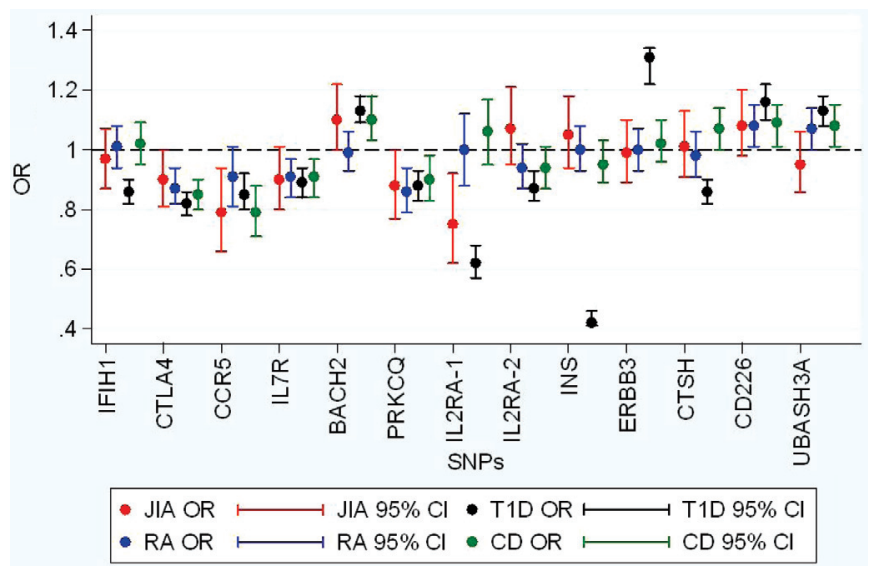

Figure 2 Odds ratio (OR) plots for juvenile idiopathic arthritis (JIA), rheumatoid arthritis (RA), type 1 diabetes (T1D) and coeliac disease (CD) for confirmed T1D loci. Data for T1D and CD from Smyth et $a{ }_{1}{ }^{2}$ for RA from Barton et $a /^{1920}$ and Eyre et al, submitted, for JIA additional data from Hinks et $a l^{56}$ and Hinks et al, 2009 in press.

Therapeutics, University of Aberdeen AB25 2ZD, UK; Pille Harrison, Paul Wordsworth: University of Oxford Institute of Musculoskeletal Sciences, Botnar Research Centre, Oxford OX3 7LD, UK.

British Society of Paediatric and Adolescent Rheumatology (BSPAR) study group $M$ Abinum, MD, M Becker, MD, A Bell, MD, A Craft, MD, E Crawley, MD, J David, MD, H Foster, MD, J Gardener-Medwin, MD, J Griffin, MD, A Hall, MD, M Hall, MD, A Herrick, MD, P Hollingworth, MD, L Holt, MD, S Jones, MD, G Pountain, MD, C Ryder, MD, T Southwood, MD, I Stewart, MD, H Venning, MD, L Wedderburn, MD, P Woo, MD, and S Wyatt, MD.

Funding This work was supported by Arthritis Research UK: AR UK grant reference No 17552. The authors would like to thank David Strachan for facilitating access to the 1958 Birth Cohort. The authors acknowledge use of genotype data from the British 1958 Birth Cohort DNA collection, funded by the Medical Research Council grant G0000934 and the Wellcome Trust grant 068545/Z/02. The authors acknowledge support from the NIHR Manchester Biomedical Research Council.

Ethics approval This study was conducted with the approval of the (North-West Multi-Centre Research Ethics Committee (MREC 99/8/84) and the University of Manchester Committee on the Ethics of Research on Human Beings).

Provenance and peer review Not commissioned; externally peer reviewed.

\section{REFERENCES}

1. Lettre G, Rioux JD. Autoimmune diseases: insights from genome-wide association studies. Hum Mol Genet 2008;17:R116-21. 
2. Smyth DJ, Plagnol V, Walker NM, et al Shared and distinct genetic variants in type 1 diabetes and celiac disease. N Engl J Med 2008;359:2767-77.

3. Coenen MJ, Trynka G, Heskamp S, et al Common and different genetic background for rheumatoid arthritis and coeliac disease. Hum Mol Genet 2009;18:4195-203.

4. Prahalad S, Shear ES, Thompson SD, et al Increased prevalence of familial autoimmunity in simplex and multiplex families with juvenile rheumatoid arthritis. Arthritis Rheum 2002;46:1851-6.

5. Hinks A, Ke X, Barton A, et al Association of the IL2RA/CD25 gene with juvenile idiopathic arthritis. Arthritis Rheum 2009;60:251-7.

6. Hinks A, Eyre S, Ke X, et al Overlap of disease susceptibility loci for rheumatoid arthritis and juvenile idiopathic arthritis. Ann Rheum Dis 2010;69:1049-53.

7. Petty RE, Southwood TR, Manners P, et al International League of Associations for Rheumatology classification of juvenile idiopathic arthritis: second revision, Edmonton, 2001. J Rheumato/ 2004:31:390-2.

8. Thomson W, Barton A, Ke X, et al Rheumatoid arthritis association at 6q23. Nat Genet 2007;39:1431-3.

9. Purcell S, Neale B, Todd-Brown K, et al PLINK: a tool set for whole-genome association and population-based linkage analyses. Am J Hum Genet 2007;81:559-75.

10. Hinks A, Barton A, John S, et al Association between the PTPN22 gene and rheumatoid arthritis and juvenile idiopathic arthritis in a UK population: further support that PTPN22 is an autoimmunity gene. Arthritis Rheum 2005;52:1694-9.

11. Albers HM, Kurreeman FA, Stoeken-Rijsbergen G, et al Association of the autoimmunity locus 4q27 with juvenile idiopathic arthritis. Arthritis Rheum 2009;60:901-4.
12. Skinningsrud B, Lie BA, Husebye ES, et al A CLEC16A variant confers risk for juvenile idiopathic arthritis and anti-CCP negative rheumatoid arthritis. Ann Rheum Dis 2009 Sep 3. [Epub ahead of print].

13. Hunt KA, Zhernakova A, Turner G, et al Newly identified genetic risk variants for celiac disease related to the immune response. Nat Genet 2008:40:395-402.

14. Garner $\mathbf{C P}$, Murray JA, Ding YC, et al Replication of celiac disease UK genome-wide association study results in a US population. Hum Mol Genet 2009;18:4219-25

15. Grunewald TG, Pasedag SM, Butt E. Cell adhesion and transcriptional activity defining the role of the novel protooncogene LPP. Trans/ Oncol 2009;2:107-16.

16. Dubé N, Cheng A, Tremblay ML. The role of protein tyrosine phosphatase 1B in Ras signaling. Proc Natl Acad Sci USA 2004:101:1834-9.

17. Fitau J, Boulday G, Coulon F, et al The adaptor molecule Lnk negatively regulates tumor necrosis factor-alpha-dependent VCAM-1 expression in endothelial cells through inhibition of the ERK1 and -2 pathways. J Biol Chem 2006;281:20148-59.

18. Prahalad S, Hansen S, Whiting A, et al Variants in TNFAIP3, STAT4, and C12orf30 loci associated with multiple autoimmune diseases are also associated with juvenile idiopathic arthritis. Arthritis Rheum 2009:60:2124-30.

19. Barton A, Eyre S, Ke X, et al Identification of AF4/FMR2 family, member 3 (AFF3) as a novel rheumatoid arthritis susceptibility locus and confirmation of two further pan-autoimmune susceptibility genes. Hum Mol Genet 2009;18:2518-22.

20. Barton A, Thomson W, Ke X, et al Rheumatoid arthritis susceptibility loci at chromosomes 10p15, 12q13 and 22q13. Nat Genet 2008;40:1156-9. 